\title{
Application of the X-FEM to the fracture of piezoelectric materials
}

Eric Béchet ${ }^{1}$, Matthias Scherzer ${ }^{2}$, Meinhard Kuna ${ }^{2}$

\author{
${ }^{1}$ LPMM-CNRS UMR 7554 \\ Université de Metz - France \\ ${ }^{2}$ IMFD - TU Bergakademie Freiberg - Germany
}




\section{Outline}

- Introduction

- Updated X-FEM formulation

- New crack tip enrichment functions

- Updated SIF computation scheme

- Use of specific preconditionner

- Convergence study

- Conclusions 


\section{Introduction}

- Goal

- Propose a updated enrichment scheme for a cracked anisotropic piezoelectric media

- Convergence study of the method

- Energy error

- SIFS and energy release rate

- Development of a SIF evaluation scheme based on interaction integrals specific to piezoelectric materials

- Numerical crack propagation using empirical laws 


\section{Introduction}

- Physical model : linear piezoelectric media, electrically impermeable crack

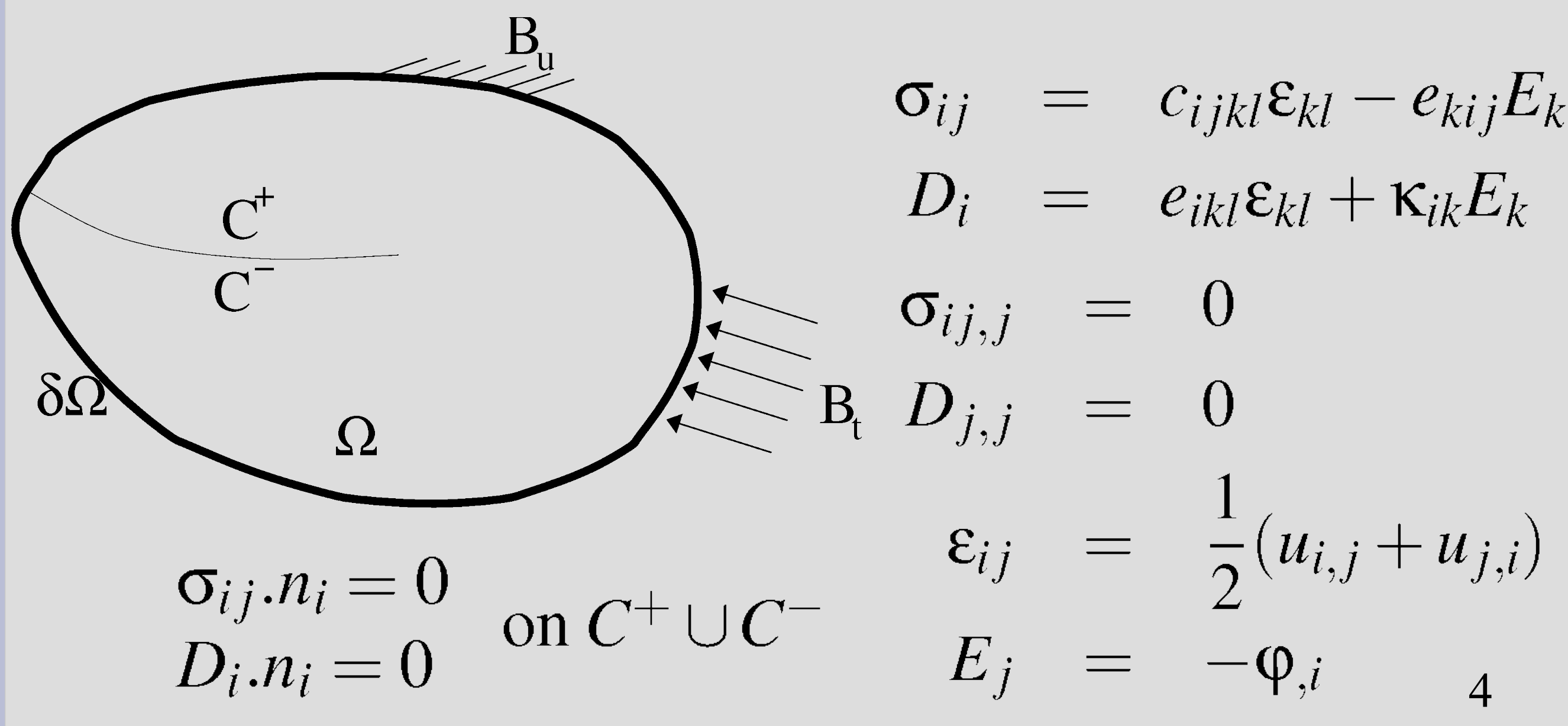




\section{Introduction}

- Numerical Model

- Xfem field approximation

- No remeshing

- Interaction integrals used to compute the SIFs 


\section{X-FEM}

- Crack represented by level-sets

- Local coodinates at the crack tip

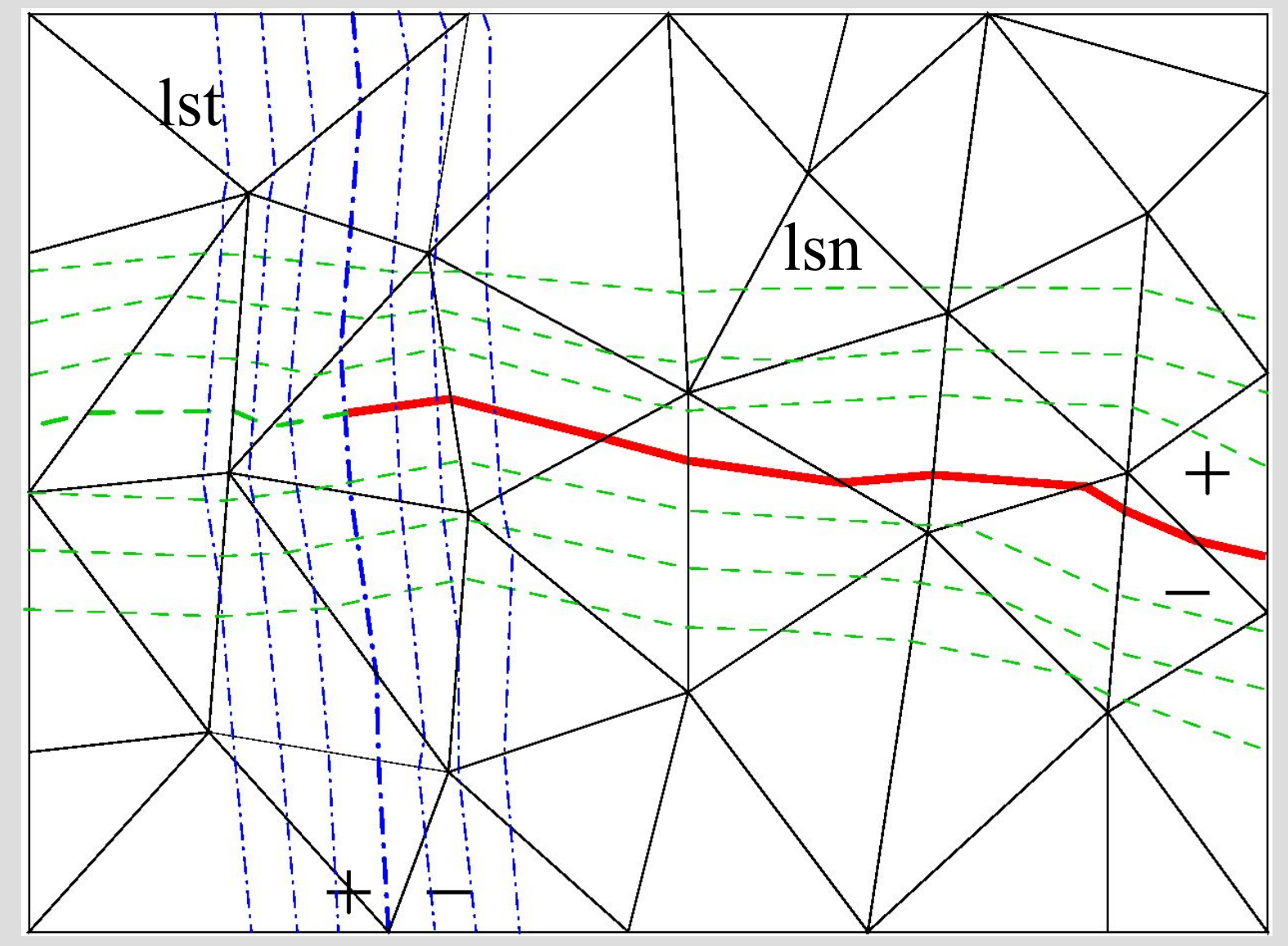




\section{X-FEM}

- Local partition of unity enrichment

- Singular functions around crack tip

- Heaviside along crack surface

- Remaining dofs unenriched

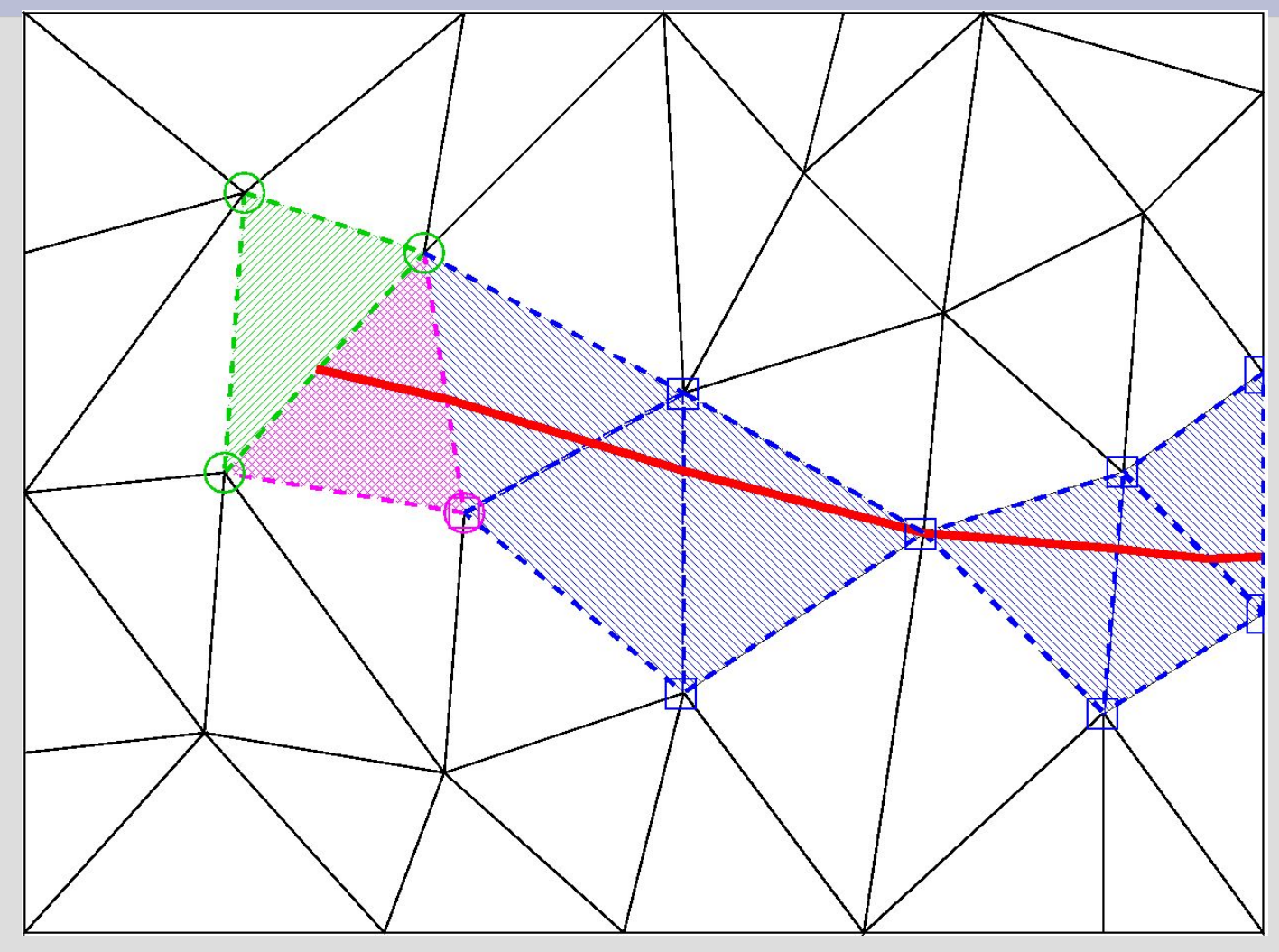

$$
\begin{aligned}
\mathbf{u}^{h} & =\sum_{i \in R} \mathbf{N}_{i} a_{i}+\sum_{i \in R j=1 \ldots n} \sum_{i} \mathbf{N}_{i} g_{j} b_{i j}+\sum_{i \in H} \mathbf{N}_{i} h c_{i} \\
\varphi^{h} & =\sum_{i \in R} N_{i} \alpha_{i}+\sum_{i \in R} \sum_{j=1 \ldots n} N_{i} g_{j} \beta_{i j}+\sum_{i \in H} N_{i} h \gamma_{i}
\end{aligned}
$$




\section{Enrichement functions}

- Enrichment functions

- Jump across the crack for displacements and potential :

$$
h(\varphi)= \begin{cases}+1 & \text { if } \varphi \geq 0 \\ -1 & \text { if } \varphi<0\end{cases}
$$

- Crack tip for in a pure mechanical setting

$g_{i}(r, \theta)=\left\{\sqrt{r} \sin \frac{\theta}{2}, \sqrt{r} \cos \frac{\theta}{2}, \sqrt{r} \sin \frac{\theta}{2} \sin \theta, \sqrt{r} \cos \frac{\theta}{2} \sin \theta\right\}$ 


\section{Enrichement functions}



\section{Enrichement functions}

- Crack tip functions for a piezoelectrical setting

- Must span the eigenfunction's space at the crack tip for displacements and potential

- Depends on the material characteristics and the orientation

- Depends on the permeability of the crack

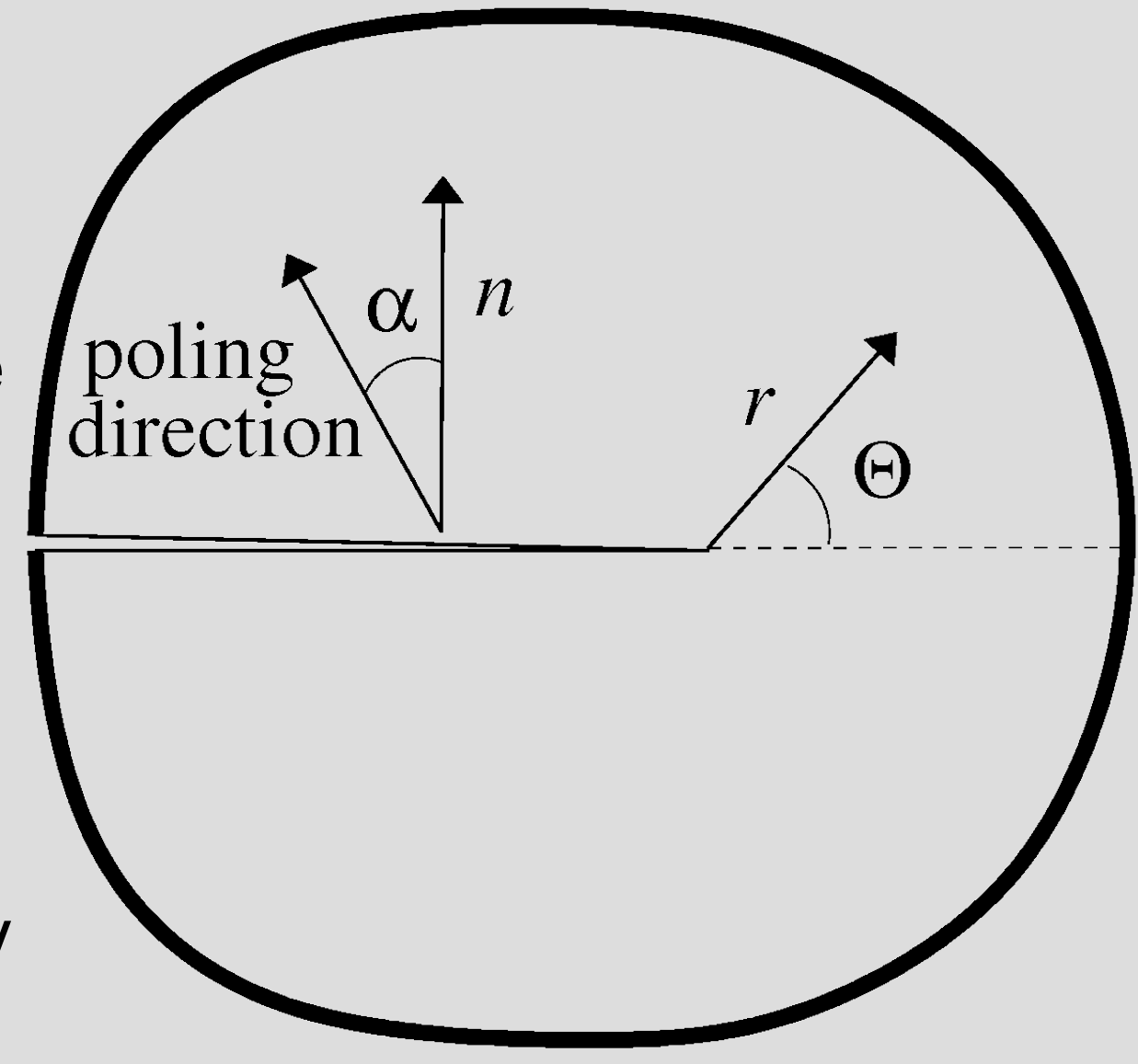

$g_{i}(r, \theta)=\left\{\sqrt{r} f_{1}(\theta), \sqrt{r} f_{2}(\theta), \sqrt{r} f_{3}(\theta), \sqrt{r} f_{4}(\theta), \sqrt{r} f_{5}(\theta), \sqrt{r} f_{6}(\theta)\right\}$ 


\section{Enrichement functions}

$f_{i}(\theta)=\phi\left(\omega(\theta, \alpha), a_{i, r e}, a_{i, i m}\right)$

$= \begin{cases}\rho\left(\omega, a_{i, r e}, a_{i, i m}\right) \cos \frac{\psi\left(\omega, a_{i, r e}, a_{i, i m}\right)}{2} & \text { if } a_{i, i m}>0 \\ \rho\left(\omega, a_{i, r e}, a_{i, i m}\right) \sin \frac{\psi\left(\omega, a_{i, r e}, a_{i, i m}\right)}{2} & \text { if } a_{i, i m} \leq 0\end{cases}$

$\omega=\theta-\alpha$

$\rho\left(\omega, a_{i, r e}, a_{i, i m}\right)=$

$\frac{1}{\sqrt{2}} \sqrt[4]{a_{i, r e}^{2}+a_{i, i m}^{2}+a_{i, r e} \sin 2 \omega-\left(a_{i, r e}^{2}+a_{i, i m}^{2}-1\right) \cos 2 \omega}$

$\psi\left(\omega, a_{i, r e}, a_{i, i m}\right)=\frac{\pi}{2}+\pi \operatorname{int}\left(\frac{\omega}{\pi}\right)$

$-\arctan \left(\frac{\cos \left(\omega-\pi \operatorname{int}\left(\frac{\omega}{\pi}\right)\right)+a_{i, r e} \sin \left(\omega-\pi \operatorname{int}\left(\frac{\omega}{\pi}\right)\right)}{\left|a_{i, i m}\right| \sin \left(\omega-\pi \operatorname{int}\left(\frac{\omega}{\pi}\right)\right)}\right)$ 


\section{Enrichement functions}

- Modified functions

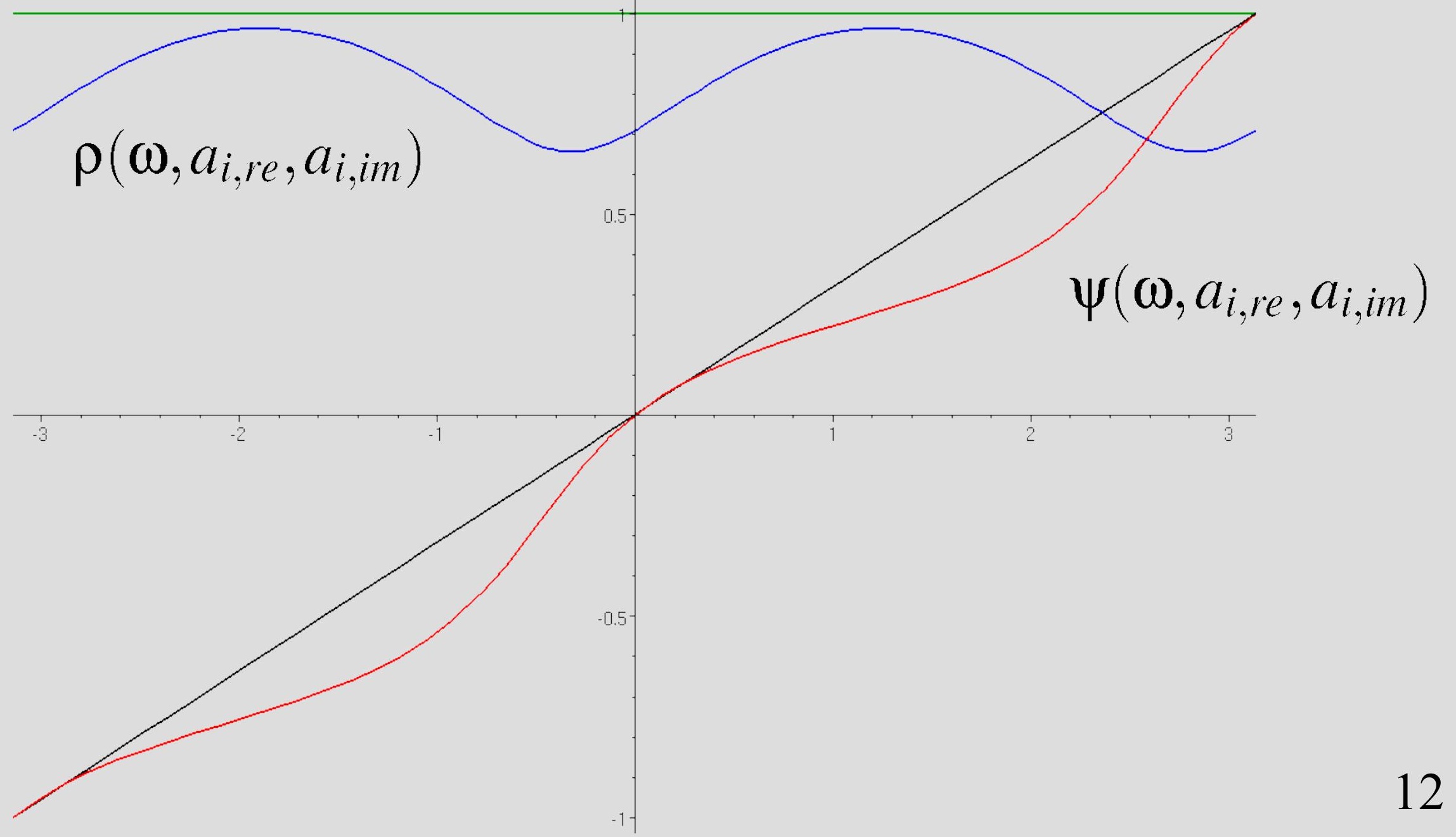




\section{Enrichement functions}



\section{Updated enrichment scheme}

"topological” Enrichment
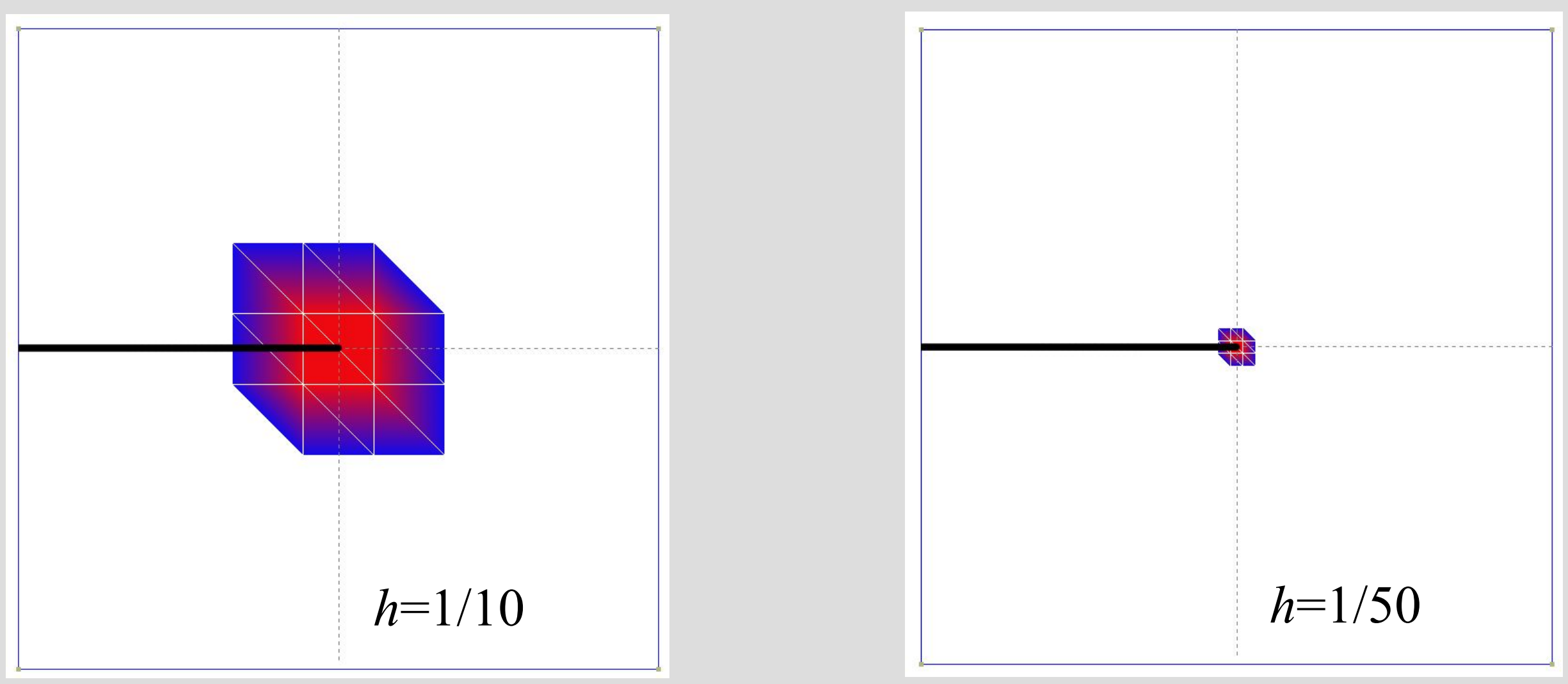


\section{Updated enrichment scheme}

\section{"Geometrical” Enrichment}
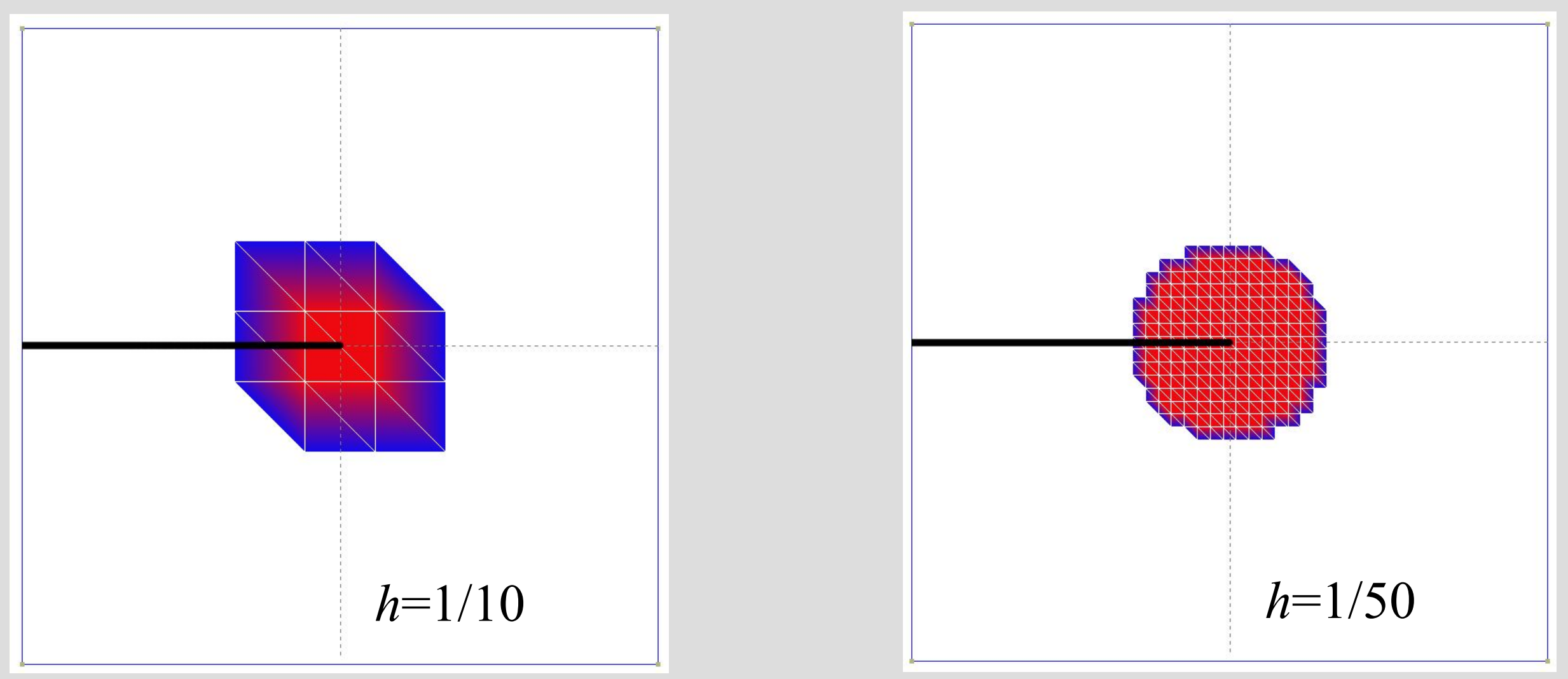


\section{Condition number}

- The enrichment may lead to almost-singular matrices $\rightarrow$ difficult to use iterative solvers






\section{Preconditionner}

- Orthogonalize each subset of enriched dofs

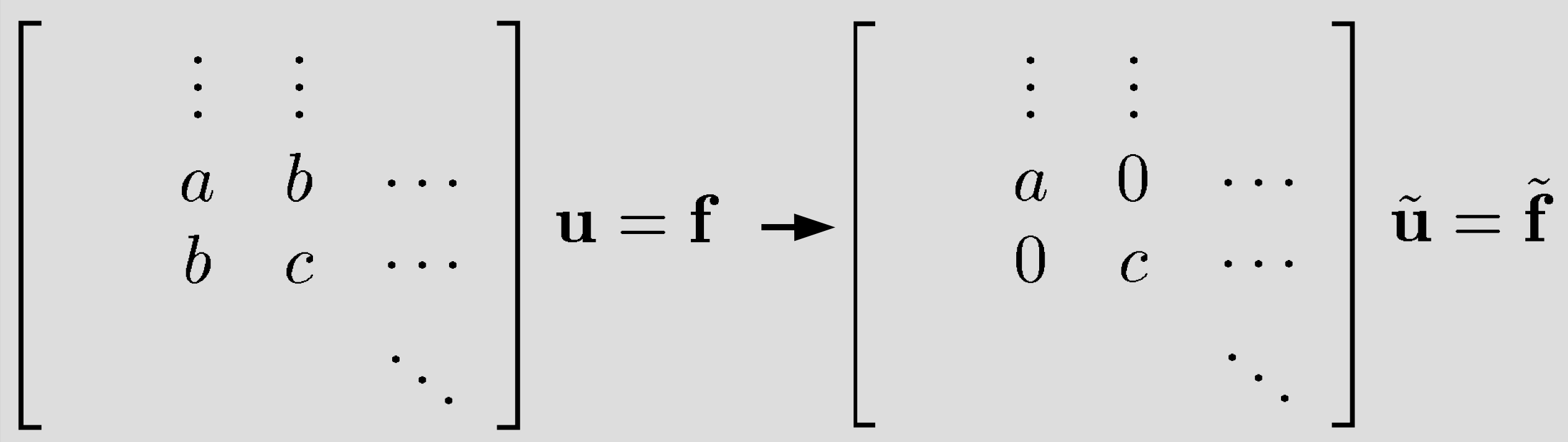




\section{Preconditionner}

- Cholesky decomposition \& scaling for node $k$ :

$$
\begin{aligned}
& \mathbf{A}=\left[\begin{array}{ll}
a & b \\
b & c
\end{array}\right] \quad \mathbf{A}=\mathbf{G G}^{T} \\
& \mathbf{D}_{i j}=\sqrt{\mathbf{A}_{i j} \delta_{i j}} \quad \text { (no summation) } \\
& \mathbf{R}=\mathbf{G}^{-1} \mathbf{D}
\end{aligned}
$$

"Assembly" of every submatrix $\mathrm{R}$ gives $\mathrm{R}^{*}$

$\mathbf{R}^{*} \mathbf{K R}^{* T} \tilde{\mathbf{u}}=\mathbf{R}^{*} \mathbf{f}$ with $\mathbf{u}=\mathbf{R}^{* T} \tilde{\mathbf{u}}$ 


\section{Preconditionner}

- Trick for handling non positive definite systems (but blockwise positive definite)

- If the matrix A belongs to the electrostatic part:

- $a, b$ and $c$ are negative

- we need to take the opposite matrix (which is positive definite) in order to generate the preconditionner

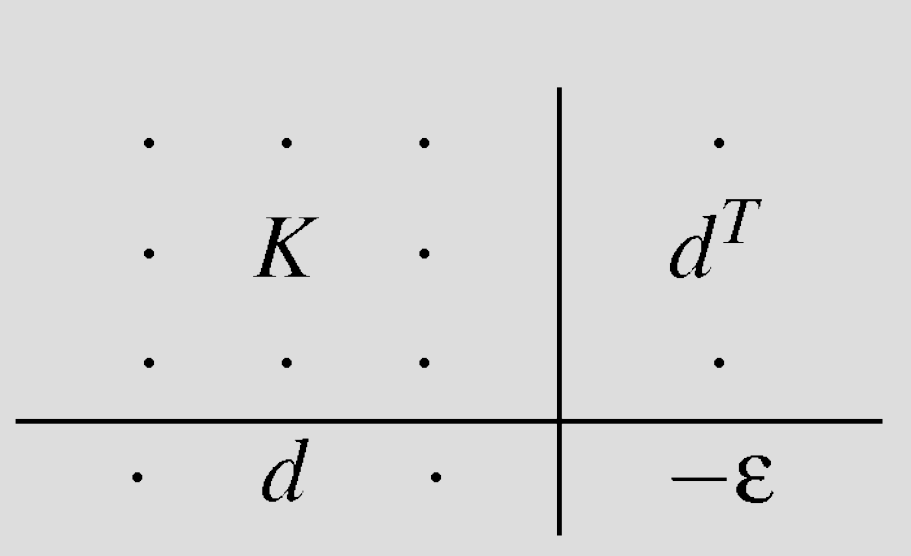

$$
\begin{gathered}
\mathbf{A}=\left[\begin{array}{cc}
-a & -b \\
-b & -c
\end{array}\right] \\
\mathbf{A}=\mathbf{G G}^{T}
\end{gathered}
$$




\section{Preconditionner}

\section{Condition number of $\mathbf{R}^{*} \mathbf{K} \mathbf{R}^{* T}$ or $\mathbf{R}^{*} \mathbf{M} \mathbf{R}^{* T}$}

\section{(Geometrical enrichment)}






\section{Convergence study}

- Exact solution - use of complex potentials

- cf. H. Sosa, Plane problems in piezoelectric media with defects, Int. J. Sol. Struct. (1991)

$$
\begin{array}{lcc}
\varepsilon_{x x}=2 \mathfrak{R}\left(\sum_{k=1}^{3}\left(a_{11} \tau_{k}^{2}+a_{12}-b_{12} \kappa_{k}\right) \phi_{k}\left(z_{k}\right)\right) & \kappa_{k}=-\frac{\left(b_{21}+b_{13}\right) \tau_{k}+b_{22}}{\delta_{11} \tau_{k}^{2}+\delta_{22}} \\
\varepsilon_{x y}=2 \Re\left(\sum_{k=1}^{3}\left(a_{12} \tau_{k}^{2}+a_{22}-b_{22} \kappa_{k}\right) \phi_{k}\left(z_{k}\right)\right) & z_{k}=x+\tau_{k} y \\
\varepsilon_{x y}=\Re\left(\sum_{k=1}^{3}\left(-a_{33} \tau_{k}+b_{13} \tau_{k} \kappa_{k}\right) \phi_{k}\left(z_{k}\right)\right) & \phi_{k}\left(z_{k}\right)=\frac{A_{k} z_{k}}{\sqrt{z_{k}^{2}-a^{2}}}+B_{k} \\
E_{x}=2 \Re\left(\sum_{k=1}^{3}\left(b_{13}+\delta_{11} \kappa_{k}\right) \tau_{k} \phi_{k}\left(z_{k}\right)\right) & \tau_{k} \text { are roots of the characteristic equation }
\end{array}
$$

$E_{y}=-2 \Re\left(\sum_{k=1}^{3}\left(b_{21} \tau_{k}^{2}+b_{22}+\delta_{22} \kappa_{k}\right) \phi_{k}\left(z_{k}\right)\right)$ 


\section{Convergence study}

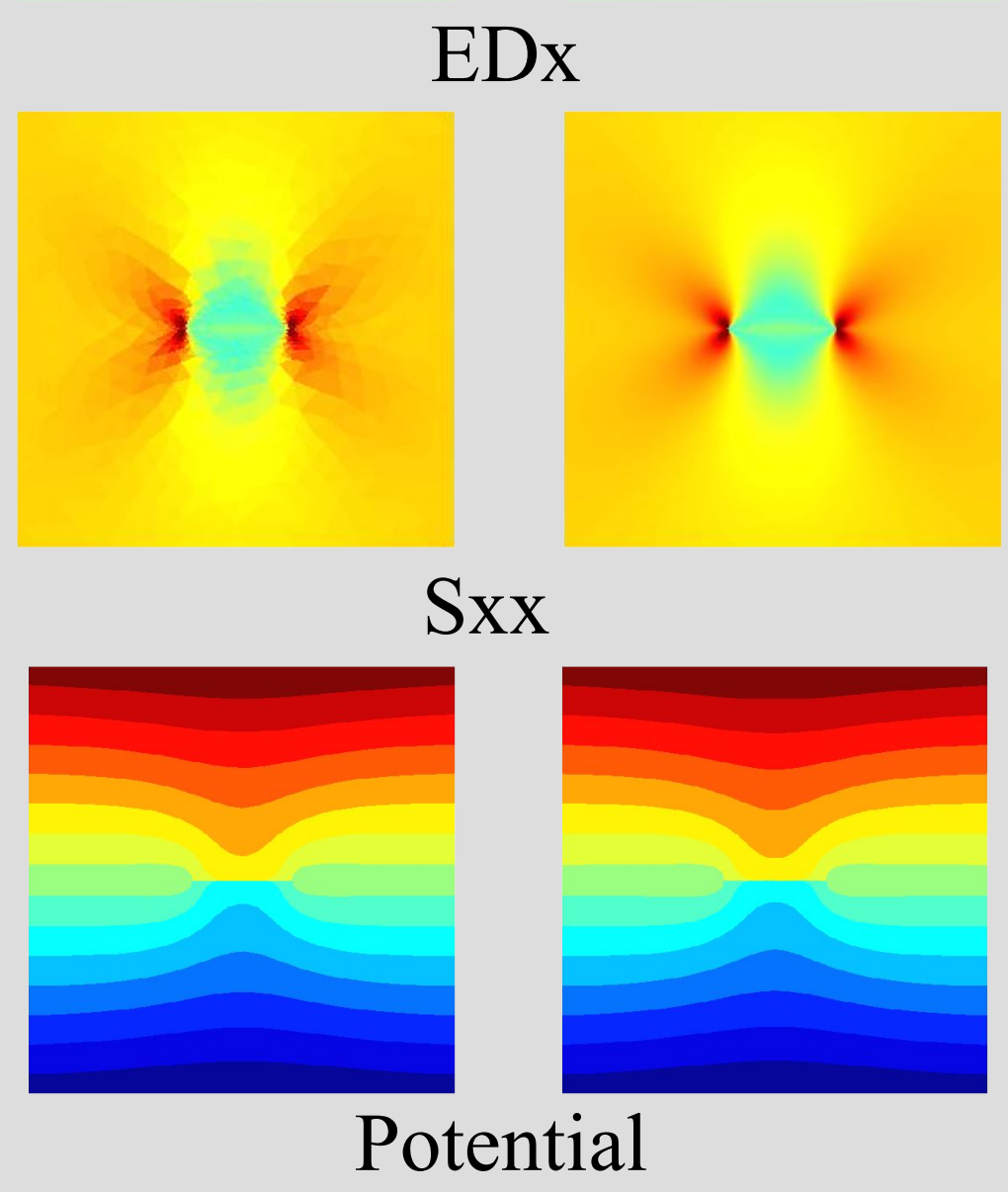

EDy

B

Sxy



B

Syy 


\section{Convergence study}
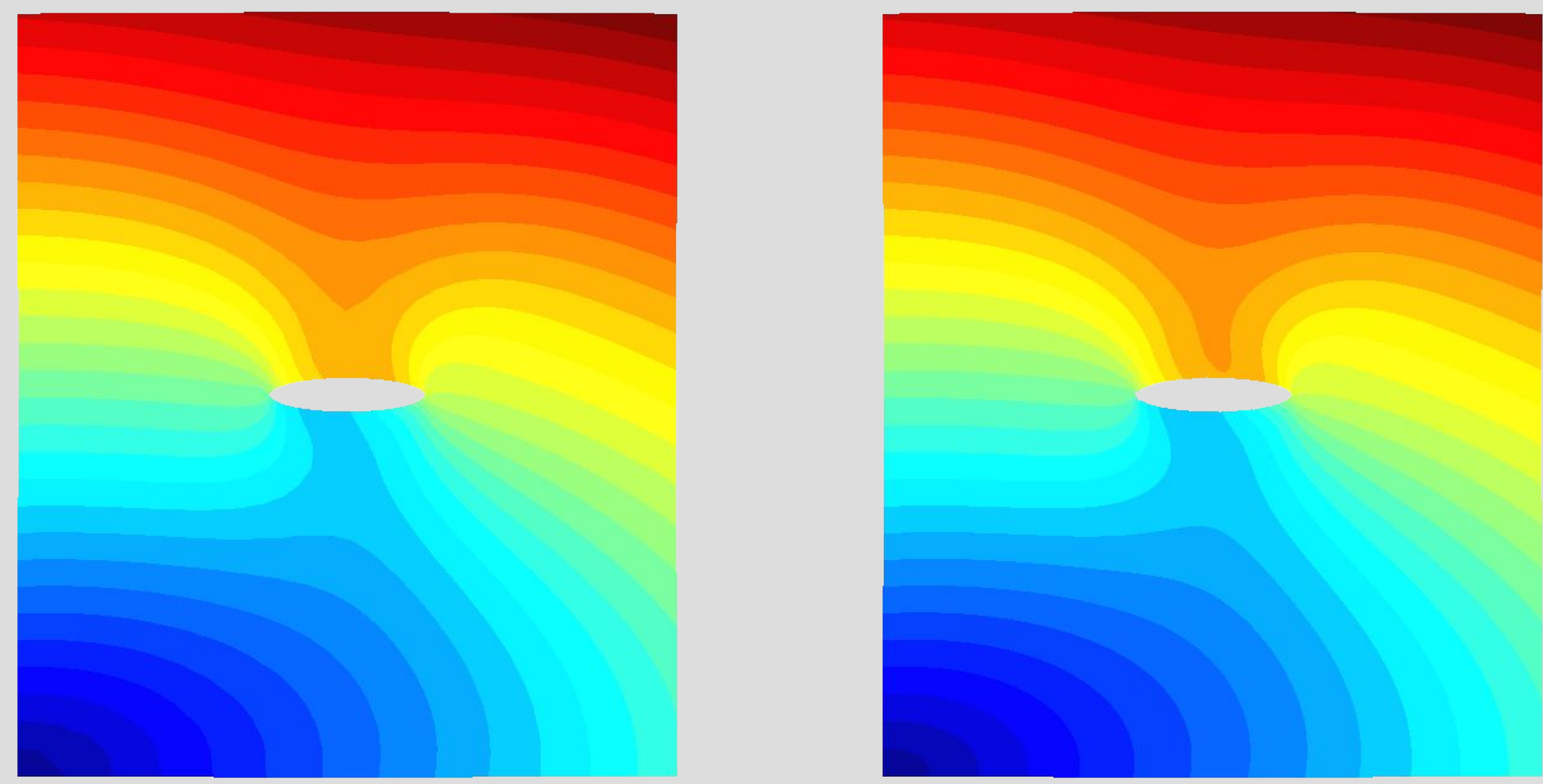

Displacement 


\section{Convergence study}

- Energy norm with respect to the internal energy

$$
U=\int_{V}\left(\frac{1}{2} \varepsilon_{i j} c_{i j k l} \varepsilon_{k l}+\frac{1}{2} E_{i} \varepsilon_{i j} E_{j}\right) d V
$$

$E_{U}=$

$\int_{V} \sqrt{\frac{1}{2}\left(\varepsilon_{i j}-\varepsilon_{i j}^{e x}\right) c_{i j k l}\left(\varepsilon_{k l}-\varepsilon_{k l}^{e x}\right)+\frac{1}{2}\left(E_{i}-E_{i}^{e x}\right) \varepsilon_{i j}\left(E_{j}-E_{j}^{e x}\right)} d V$

$=$

$\int_{V} \sqrt{\frac{1}{2}\left(u_{i}-u_{i}^{e x}\right)_{, j} c_{i j k l}\left(u_{k}-u_{k}^{e x}\right)_{, l}+\frac{1}{2}\left(\varphi-\varphi^{e x}\right)_{, i} \varepsilon_{i j}\left(\varphi-\varphi^{e x}\right)_{, j}} d V$ 


\section{Convergence study}

- Energy norm

- comparison with standard crack tip enrichment

- Infinite body with embedded crack

- inclined material axes $\left(30^{\circ}\right)$

- PZT4 orthotropic material

$$
\begin{aligned}
& c=10^{10}\left[\begin{array}{cccccc}
14.02 & 7.892 & 7.565 & 0 & 0 & 0 \\
7.892 & 14.02 & 7.565 & 0 & 0 & 0 \\
7.565 & 7.565 & 11.58 & 0 & 0 & 0 \\
0 & 0 & 0 & 2.527 & 0 & 0 \\
0 & 0 & 0 & 0 & 2.527 & 0
\end{array}\right] \quad e=10^{0}\left[\begin{array}{cccccc}
0 & 0 & 0 & 0 & 13.0 & 0 \\
0 & 0 & 0 & 13.0 & 0 & 0 \\
-5.268 & -5.268 & 15.44 & 0 & 0 & 0
\end{array}\right] \\
& \varepsilon=10^{-9}\left[\begin{array}{ccc}
6.68 & 0 & 0 \\
0 & 6.68 & 0 \\
0 & 0 & 5.523
\end{array}\right]
\end{aligned}
$$




\section{Energy error}

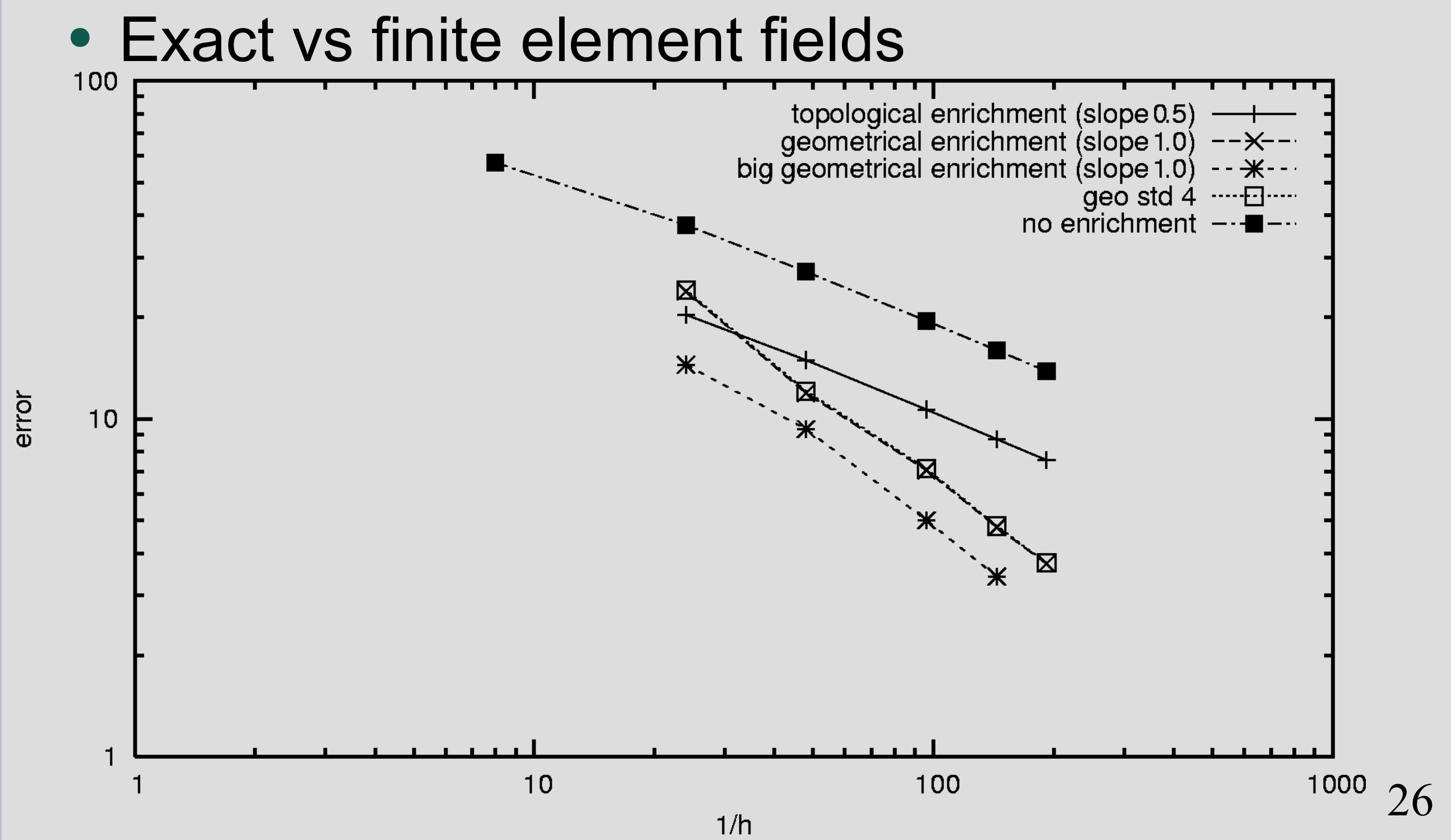




\section{Energy error}

- The "classical" enrichment gives almost the same results as the specific enrichment, with less computational overhead.

- It is not clear whether different material laws (e.g. "more" anisotropic) lead to different results

$g_{i}(r, \theta)=\left\{\sqrt{r} \sin \frac{\theta}{2}, \sqrt{r} \cos \frac{\theta}{2}, \sqrt{r} \sin \frac{\theta}{2} \sin \theta, \sqrt{r} \cos \frac{\theta}{2} \sin \theta\right\}$ 


\section{SIFs computation}

- Contour integrals on $\Gamma \rightarrow$ Domain integrals over $\mathrm{V}$

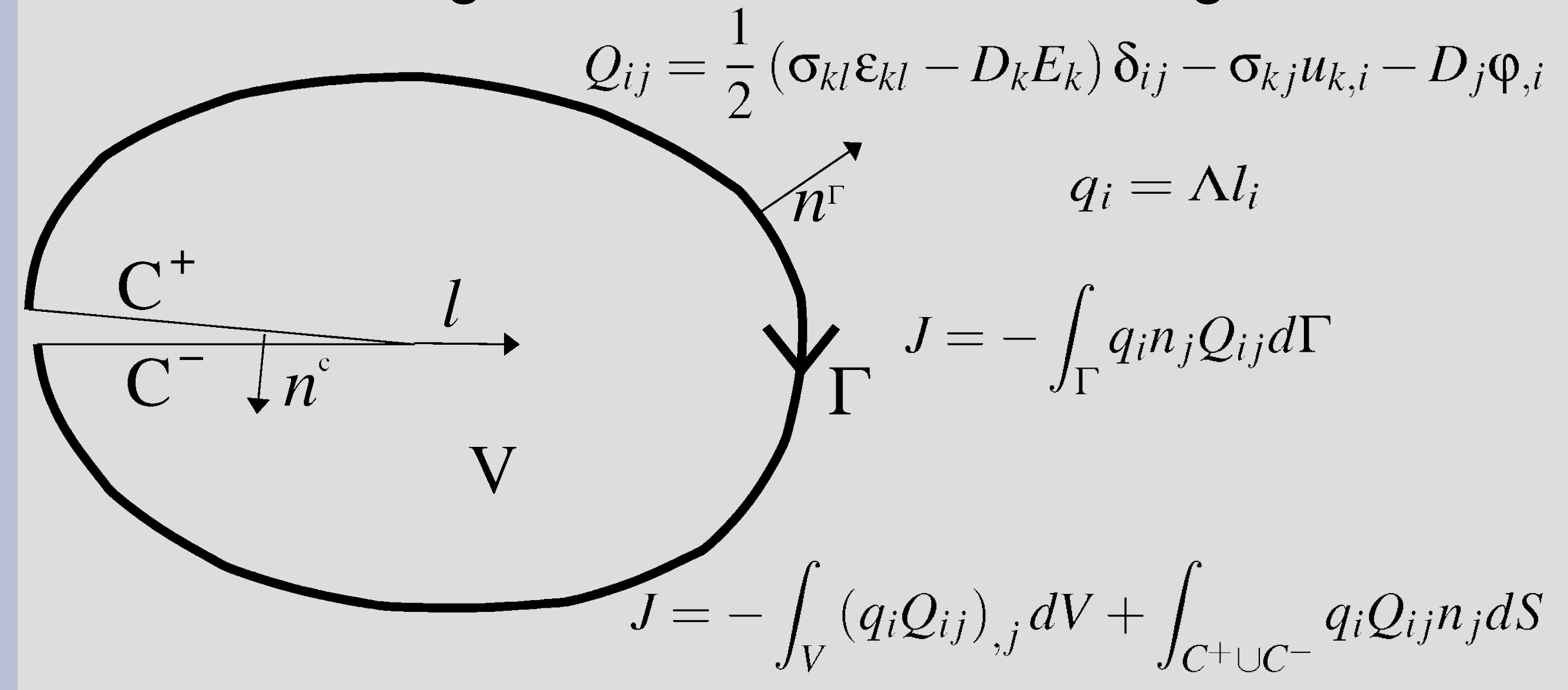




\section{Interaction integrals}

- Same procedure used to compute interaction integrals (no crack loading) :

$$
\begin{aligned}
I= & \int_{V} q_{i, j}\left(\sigma_{k j} u_{k, i}^{a u x}+\sigma_{k j}^{a u x} u_{k, i}+D_{j} \varphi_{, i}^{a u x}+D_{j}^{a u x} \varphi_{, i}\right) d V \\
& +\int_{V} q_{i}\left(\sigma_{k j} u_{k, i j}^{a u x}+\sigma_{k j, j}^{a u x} u_{k, i}+D_{j} \varphi_{, i j}^{a u x}+D_{j, j}^{a u x} \varphi_{, i}\right) d V \\
& -\int_{V} q_{i, j}\left(\sigma_{k l} \varepsilon_{k l}^{a u x}-D_{k} E_{k}^{a u x}\right) \delta_{i j} d V \\
& -\int_{V} q_{i}\left(\sigma_{k l} \varepsilon_{k l, i}^{a u x}-D_{k} E_{k, i}^{a u x}\right) d V
\end{aligned}
$$

$I=\int_{\Gamma} G^{a u x} \Lambda d \Gamma \quad G^{a u x}=Y_{M N} K_{M} K_{N}^{a u x} \quad Y_{M N} K_{M} K_{N}^{a u x}=\frac{I}{\int_{\Gamma} \Lambda d \Gamma}$ 


\section{Interaction integrals}

- Relation between $G$ and the $K$ factors

- Simpler case of the isotropic elasticity well known

$Y_{M N} K_{M} K_{N}^{a u x}=\left\{K_{I} K_{I I} K_{I I I}\right\}\left[\begin{array}{ccc}\frac{2\left(1-v^{2}\right)}{E} & 0 & 0 \\ 0 & \frac{2\left(1-v^{2}\right)}{E} & 0 \\ 0 & 0 & \frac{2(1+v)}{E}\end{array}\right]\left\{\begin{array}{c}K_{I}^{\text {aux }} \\ K_{I I}^{a u x} \\ K_{I I I}^{a u x}\end{array}\right\}$

- The Irwin matrix depends on the material orientation and is not explicitely known for piezos.

$$
G^{a u x 1, a u x 2}=Y_{M N} K_{M}^{a u x 1} K_{N}^{a u x 2}
$$

$I^{a u x 1, a u x 2}=\int_{\Gamma} G^{a u x 1, a u x 2} \Lambda d \Gamma \quad Y_{M N} K_{M}^{a u x 1} K_{N}^{a u x 2}=\frac{I^{a u x 1, a u x 2}}{\int_{\Gamma} \Lambda d \Gamma}$ 


\section{Interaction integrals}

- By using the eigenfunction set, every term in the Irwin matrix can be determined

- for instance : $\quad\left\{K_{I}^{a u x 1}=1 K_{I I}^{a u x 1}=0 K_{I I I}^{a u x 1}=0 K_{I V}^{a u x}=0\right\}$

$$
\left\{K_{I}^{a u \times 2}=0 K_{I I}^{a u x 2}=1 K_{I I I}^{a u x 2}=0 K_{I V}^{a u x 2}=0\right\}
$$

$I^{\text {aux } x, \text { au } x 2}$

$\frac{I_{\Gamma} \Lambda d \Gamma}{\int_{\Gamma}}=Y_{M N} K_{M}^{\text {auxl }} K_{N}^{\text {aux } 2}$

$$
=Y_{12}=Y_{21}
$$

- No need of finite element support because the Irwin matrix is intrinsic (for a given material orientation)

$I^{a u x 1, a u x 2}=\int_{\Gamma} q_{i} n_{j}\left(\sigma_{k j}^{a u x 1} u_{k, i}^{a u x 2}+\sigma_{k j}^{a u x 2} u_{k, i}^{a u x 1}+D_{j}^{a u x 1} \varphi_{, i}^{a u x 2}+D_{j}^{a u x 2} \varphi_{, i}^{a u x 1}\right) d \Gamma$

$$
-\int_{\Gamma} q_{i} n_{j} \frac{1}{2}\left(\sigma_{k l}^{a u x 1} \varepsilon_{k l}^{a u x 2}+\sigma_{k l}^{a u x 2} \varepsilon_{k l}^{a u x 1}-D_{k}^{a u x 1} E_{k}^{a u x 2}-D_{k}^{a u x 2} E_{k}^{a u x 1}\right) \delta_{i j} d \Gamma
$$




\section{Choice of $\Lambda$}

The field $\Lambda$ describes the geometry of the integration domain $S$.

$$
\Lambda=\sum_{i} N_{i} \Lambda_{i} \quad \Lambda_{i}= \begin{cases}1 & \text { if support }\left(N_{i}\right) \subset S \\ 0 & \text { otherwise }\end{cases}
$$

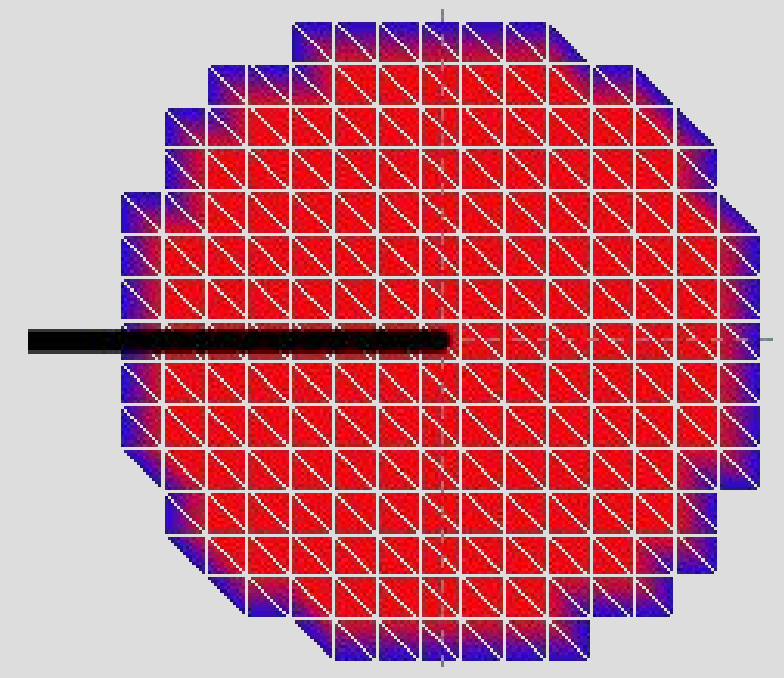

Two choices of integration domain with regard to $h$ :

- Topological

- Geometrical 


\section{$\mathrm{J}$ error}

- Exact J vs computed J-integral

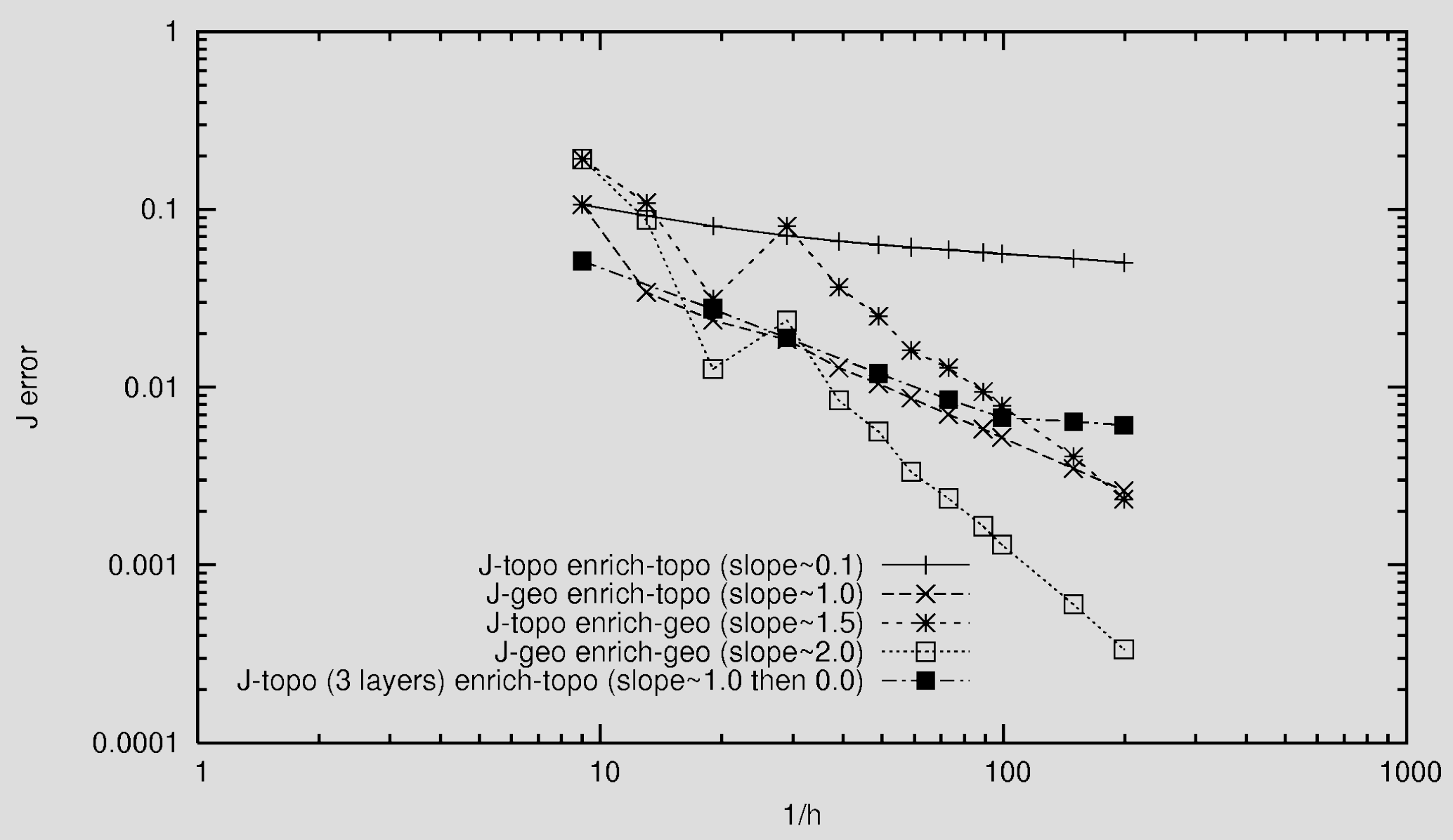




\section{Conclusion \& future work}

- Application of the X-Fem for piezos

- The convergence study shows that the four classical enrichment functions are enough

- Use of equivalement volume integrals to compute the electromechanical J-integral

- Interaction integrals will be used to extract K factors

- Auxiliary fields can also be used to compute the local Irwin matrix 


\section{Conclusion \& future work}

- Systematic investigations of $\mathrm{J}$ and the $\mathrm{K}$ factors's accuracy

- Propagation laws

- Investigation for a electrically permeable crack

- 3D extensions (esp. for the eigenfunctions needed for K extraction) 